\title{
THE RECEPTOR POTENTIAL AND ADAPTATION IN THE COCKROACH TACTILE SPINE ${ }^{1}$
}

\author{
A. S. FRENCH
}

\author{
Department of Physiology, University of Alberta, Edmonton, Alberta, T6G $2 \mathrm{H} 7$ Canada
}

Received October 19, 1983; Revised February 15, 1984; Accepted March 21, 1984

\begin{abstract}
The receptor potential in the cockroach tactile spine was measured during mechanotransduction by recording from the afferent axon about $0.5 \mathrm{~mm}$ from the sensory ending. The receptor potential was a linear function of spine position, but its amplitude and phase varied with the mean position of the spine. This can be related to the mechanical linkage from the socket of the spine to the sensory ending.

The frequency response for mechanotransduction was flat over a frequency range of 0.1 to $100 \mathrm{~Hz}$, after accounting for the cable properties of the axon, and there was no evidence of adaptation. The length constant and membrane time constant of the axon were estimated to be $130 \mu \mathrm{m}$ and $1 \mathrm{msec}$, respectively.

The threshold amplitude of movement required to produce action potentials was also measured as a function of frequency. It gave a power law relationship which was exactly the inverse of the frequency response of the receptor during strong stimulation and rapid tiring. This suggests that adaptation of the sensory discharge in this receptor is produced by the action potential encoding mechanism.
\end{abstract}

Many mechanoreceptors exhibit the phenomenon of adaptation, which may be defined as a decrease in response with time following a step increase in the input stimulus. In the frequency domain this corresponds to an increasing sensitivity with increasing frequency. Mechanotransduction is conventionally viewed as a three-stage process: (1) the stimulus is coupled mechanically to the sensitive membrane of the receptor; (2) the deformation is transduced into an electrical signal, the generator potential or receptor potential; (3) the receptor potential is encoded into a train of action potentials for transmission to the central nervous system (Eyzaguirre and Kuffler, 1955; Loewenstein, 1959). Adaptation could occur at any stage of the process.

Mechanical processes have been implicated in the adaptation of the slowly and rapidly adapting crayfish stretch receptors (Eyzaguirre and Kuffler, 1955; Brown and Stein, 1966), and their contribution to the total adaptation of the slowly adapting receptor was estimated to be $70 \%$ (Nakajima and Onodera, 1969a). In frog skin receptors the mechanical components appear to be important (Loewenstein, 1956), and a viscoelastic model has been proposed to account for adaptation in frog and rat skin receptors (Catton and Petoe, 1966). Mechanical adaptation has been demonstrated in the Pacinian corpuscle, where adaptation in the receptor potential was reduced by removing part of the mechanical structure (Mendelson and Loewenstein, 1964). In insect cuticular mechanoreceptors viscoelastic properties have been reported to be significant in campaniform sensilla (Chapman et al., 1979) and in hair sen-

\footnotetext{
${ }^{1}$ This work was supported by grants from the Medical Research Council of Canada and the Alberta Heritage Foundation for Medical Research. I would like to thank Rodney Gramlich for expert technical assistance throughout.
}

silla (Buno et al., 1981). Direct observation of viscous creep has been made in mammalian muscle spindles (Boyd et al., 1977) and the receptor potential in muscle spindles has been reported to show significant adaptation (Hunt and Wilkinson, 1980). However, some adaptation in the crayfish stretch receptors and muscle spindles has been attributed to changes in the potassium conductance of the membrane or activity of the sodium-potassium pump, which could directly affect the amplitude of the receptor potential (Nakajima and Onodera, 1969a; Husmark and Ottoson, 1971a, b; Ottoson, 1974).

The action potential encoding process has been linked to adaptation in several receptors. The difference between the slowly and rapidly adapting crayfish stretch receptors has been attributed to accommodation to a rising generator potential in the rapidly adapting receptor (Nakajima and Onodera, 1969b), and the same conclusion has been reached regarding the difference between slowly and rapidly adapting amphibian skin receptors (Vallbo, 1964a). Removal of the lamellar layers from the Pacinian corpuscle reduced adaptation in the receptor potential, but the receptor would still produce only one action potential in response to a step deformation (Mendelson and Loewenstein, 1964). In the muscle spindle there was greater adaptation in the action potential discharge (Matthews and Stein, 1969) than in the receptor potential (Hunt and Wilkinson, 1980). Therefore, it seems that mechanical components or ionic changes can contribute to adaptation of the receptor potential, but rapid adaptation usually occurs in the encoding process. However, the number of mechanoreceptors in which it has been possible to separate the processes by observing the receptor potential is stiil small.

The mechanoreceptor used in the present work was the femoral tactile spine of the cockroach. This is a typical insect cuticular receptor (McIver, 1975) with a bipolar sensory cell in 
the periphery having a single sensory dendrite containing a specialized structure called the tubular body (French and Sanders, 1981). Transduction between movement and afferent action potentials in this sensillum has been studied a number of times (Chapman and Smith, 1963; French et al., 1972; French, 1980; French and Kuster, 1981). It is rapidly adapting, and its response to deterministic and random inputs can be well fitted by a power law or fractional differentiator model. Fractional differentiation, which in a mechanoreceptor corresponds to a response between position sensitivity and velocity sensitivity, has been used to characterize the adaptation of a number of different sensory receptors (Thorson and Biederman-Thorson, 1974), and it is commonly found in insect cuticular mechanoreceptors (Bohnenberger, 1981). In view of the complexity of the tubular body structure and the known contribution of mechanical components to adaptation in other receptors, we have previously suggested that the viscoelastic properties of the tubular body might contribute to the dynamic response of the tactile spine and other cuticular receptors (French and Sanders, 1981; French and Kuster, 1982).

Although cuticular mechanoreceptors have been widely studied both morphologically and electrophysiologically, it has so far been impossible to record their receptor potentials intracellularly. Ilowever, I have now measured the receptor potential in the tactile spine by extracellular recording of the decrementally conducted voltage in the sensory axon. The receptor potential was a linear representation of the spine position for small movements, and the frequency response between movement and receptor potential was flat, after accounting for the cable properties of the axon. Therefore, adaptation in the receptor probably occurs during the encoding process.

\section{Materials and Methods}

The experimental approach was to measure the receptor potential in the electrically isolated axon a short distance from the sensory ending. This technique has previously been used to examine the receptor potential in several mechanoreceptors, including the muscle spindle (Katz, 1950; Hunt and Wilkinson, 1980) and the Pacinian corpuscle (Loewenstein, 1959).

Cockroaches, Periplaneta americana, were maintained in a laboratory colony. The experimental arrangement for stimulating the tactile spine and recording the receptor potential is illustrated in Figure 1. Experiments were performed on metathoracic legs of adult animals. The leg was amputated at the femur, distal to the trochanter, and was waxed onto a cork base. Care was taken to ensure that the main trachea in the femur was open and exposed to air, since otherwise the response failed rapidly from anoxia. The electromagnetic pusher and the position transducer have been described in detail before (French and Kuster, 1981). The afferent axon from the spine runs into nerve 5 via a fine branch, not described before, which I have called $5 \mathrm{r} 8 \mathrm{e}$ by the standard nomenclature (Nijenhuis and Dresden, 1952). This branch was picked up into a paraffin oil bath by a platinum hook electrode as close as possible to the spine. The distance from the electrode to the spine was in the range of 500 to $1000 \mu \mathrm{m}$. A reference platinum electrode was inserted into the femur through the cut end. Both platinum electrodes were treated by electrolysis in a solution of $3 \%$ platinum chloride to reduce low frequency noise in the recording. The receptor potential was recorded by a DC amplifier of conventional design but using low noise integrated circuits throughout. To reduce noise and cross-talk between the stimulating and recording electronics there was electrical shielding around the electromagnetic pusher and the isolated leg. The signal from the position detector and the receptor potential were fed to separate analogue to digital converters connected to a PDP-11 computer.

Noise levels and cross-talk figures were measured over the frequency range used for the experiments. To approximate the experimental conditions as closely as possible the measurements were made with the electrode picking up an adjacent nerve which did not carry the sensory axon, or with the correct nerve but with the pusher not waxed to the spine. The noise in the receptor potential increased with decreasing frequency as is normally found in electronic and electrophysiological

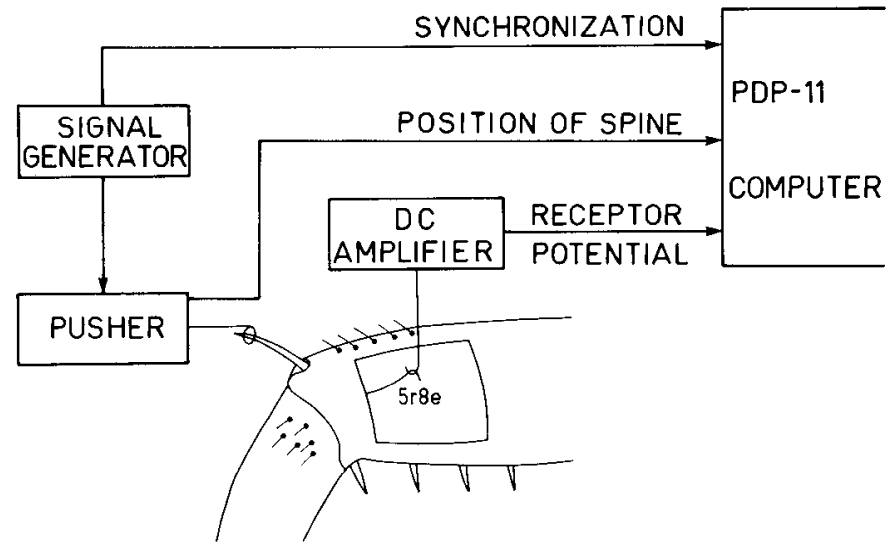

Figure 1. The experimental arrangement for recording the receptor potential. The pusher consisted of a small loudspeaker modified to push a glass rod which was waxed to the tip of the tactile spine. The position of the rod was sensed by an infrared phototransistor mounted on the loudspeaker and illuminated by a fixed infrared light source. The position signal was used to complete a servo loop control of loudspeaker position with appropriate feedback compensation. The pusher servo was driven by a signal generator which also provided a synchronizing pulse to the computer at the start of each cycle. The sensory nerve from the spine was picked up by a platinum hook electrode into a paraffin oil bath surrounded by a Vaseline dam. The receptor potential, relative to another platinum electrode in the femur, was amplified by a low noise DC amplifier before being fed to the computer via an analogue to digital converter. The position signal was fed to the computer by a separate but identical converter.

measurements. At the lowest frequency used of $0.1 \mathrm{~Hz}$ and after taking 100 averages of the signal, the average noise level was $330 \mathrm{nV}$. By comparison the receptor potential at this frequency was typically 5 to $50 \mu \mathrm{V}$. Cross-talk between the stimulating and recording systems was reduced by isolating and shielding but could still be detected due to electromagnetic radiation from the pusher to the recording electrode. Unlike the noise signal, cross-talk increased with increasing frequency and could not be reduced by averaging. At the highest frequency used of $100 \mathrm{~Hz}$ a stimulus of $4 \mu \mathrm{m}$ peak-to-peak produced a cross-talk signal of $100 \mathrm{nV}$.

The pusher was driven with sinusoidal stimuli from a function generator (Hewlett-Packard 3310A). It was necessary to average the response to repeated cycles to obtain smooth results. For this purpose the synchronizing pulse from the function generator was fed to the PDP-11 computer via a Schmitt trigger. The computer averaged both the position transducer signal and the receptor potential signal for a requested number of cycles and then calculated the best fitting sinusoid for each of the two averages using a minimum square error procedure. The amplitudes of the best fitting sinusoids were printed out together with the gain and phase shift obtained by dividing the receptor potential by the position stimulus. To obtain the frequency response this process was repeated at each frequency of interest. Typically 100 averages were used at low frequencies and up to 5000 averages at high frequencies.

Because of the sensitivity of the receptor the preparation was mounted on an air-driven vibration isolation table (Backer-Loring micro-G). To prevent desiccation of the leg, exposed tissues at the cut end of the femur were kept moist by the application of a cockroach saline solution (Chesler and Fourtner, 1981).

\section{Results}

The tactile spine is most sensitive to movements along its long axis toward the socket (French, 1980), and this mode of stimulation was used throughout. Movements of a few micrometers were sufficient to elicit action potentials, which were recorded with an amplitude of about $20 \mathrm{mV}$ using the recording technique described here. Conventionally the action potentials in a mechanoreceptor are suppressed using tetrodotoxin (TTX) so that the reccptor potential can be observed. However, in the tactile spine the sensory dendrite and soma are embedded in a 
complex cuticular structure, and there appears to be a substan tial barrier to the passage of chemicals between the interstitial fluid and the sensory ending (Bernard et al., 1980). Application of a $10^{-6} \mathrm{M}$ TTX solution to the interstitial fluid as close as possible to the sensillum caused a rapid loss of local action potentials in the axon. However, attenuated action potentials, presumably occurring close to the sensory dendrite and being conducted decrementally along the axon, could be observed for periods of an hour or more. Even after all indication of action potential activity had ceased, it was sometimes possible to observe a frequency response function for transduction which was identical to those reported from studies on action potential signals (French, 1980). A possible cause of this observation is a calcium component in the action potentials, as has been reported in the frog muscle spindle (Ito and Komatsu, 1979). This suggests that studies on receptors which have been treated with TTX should be interpreted with caution, since small regenerative signals of approximately the same size as the receptor potential might still be contributing to the observed output. Because of these difficulties, all of the results presented here were obtained from experiments in which the movement was subthreshold and without TTX application.

The properties of the receptor potential. The response to a sinusoidal movement of the spine was a sinusoidal receptor potential, but the amplitude and phase of this response depended upon the mean position of the spine in its socket. This is illustrated in Figure 2 where the potential elicited by $1-\mathrm{Hz}$ sinusoid is shown for the same spine but at three mean posi-

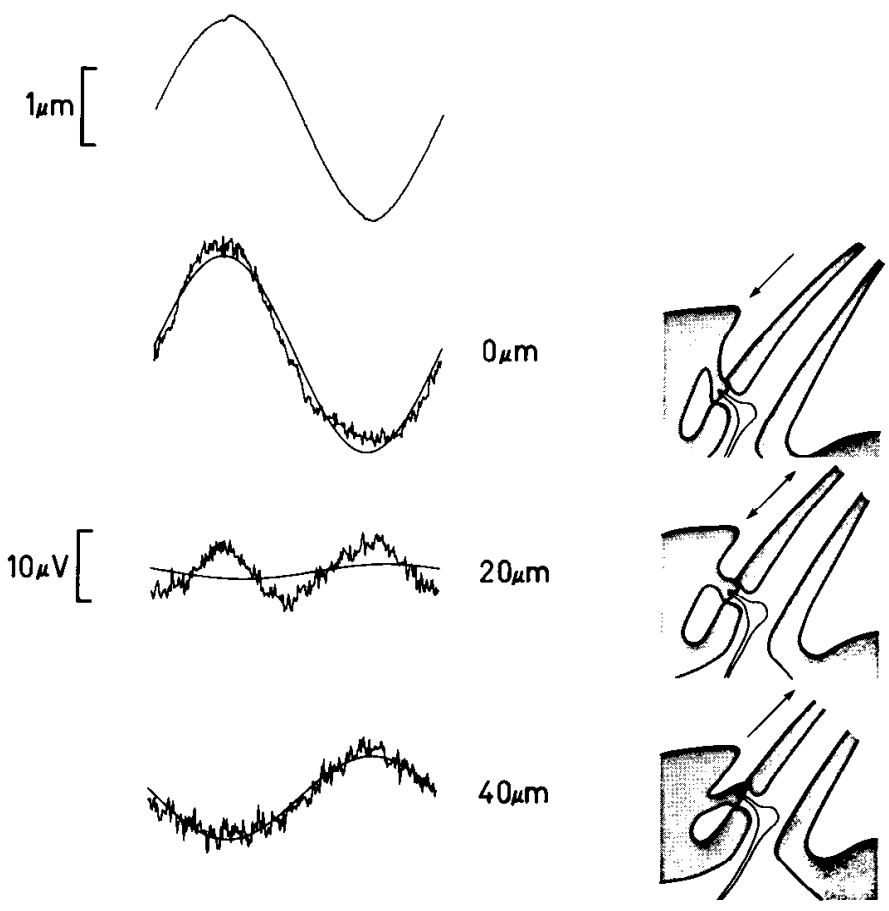

Figure 2. The averaged position and resulting receptor potentials during sinusoidal stimulation of the tactile spine at a frequency of 1 $\mathrm{Hz}$. The amplitude and phase of the receptor potential were strongly dependent upon the mean position of the spine. When it was pushed inward the response was in phase with the stimulus. When the spine was withdrawn from the first position by $20 \mu \mathrm{m}$, the response peaked twice during each cycle at the positions of maximum push and maximum pull. When the spine was withdrawn a further $20 \mu \mathrm{m}$, the response was $180 \%$ out of phase with the stimulus. The cause of these changes is believed to lie in the mechanical arrangement of the socket attachment which pulls the end of the sensory dendrite, as illustrated by the three diagrams to the right of the receptor potentials. Arrows indicate directions of movement which caused positive (depolarizing) changes in the receptor potential. tions. When the spine was pushed inward, the response was in phase with the stimulus. However, if the spine was pulled outward, the response declined in amplitude and a second peak appeared at $180^{\circ}$ phase shift to the original. With continued withdrawal of the spine this second peak predominated and the response became a simple sinusoid again, but of opposite sign to the original response and the stimulus. This behavior is believed to arise from the morphology of the sensory ending and its surrounding tissues. The sensory ending terminates in a cuticular plug which is connected to the outside socket by a structure called the socket attachment (French and Sanders, 1981). Thus, tension in this attachment leads to sensory transduction by pulling on the terminal plug, which presumably distorts the sensory ending. Tension in the attachment may be produced by pushing the spine inward or by pulling it outward, as is illustrated in Figure 2. However, the former is a more effective stimulus because the suspension system of the spine allows it to move further inward than outward from its resting position. Since positive displacement was chosen to be inward for these experiments, the pushed-in condition produced a response in phase with the stimulus, while the pulled-out condition gave an inverted response. In the intermediate state there was tension produced in the attachment when the spine was moved inward or outward, and this caused a peak in response at both extremes of movement, giving an apparent frequency doubling. Under normal conditions the spine is probably at the position of minimum tension in the socket attachment or slightly inward from it. Movements outward from this position are unnatural and require much more force on the spine than do inward movements. In all subsequent experiments the spine was pushed inward until the largest amplitude sinusoid caused a just subthreshold response.

The amplitude of the receptor potential was a linear function of spine movement. Figure 3 shows the results of an experiment in which the mean position of the spine was held constant while the amplitude of a $1-\mathrm{Hz}$ stimulating movement was varied. The maximum observable amplitude was limited by the

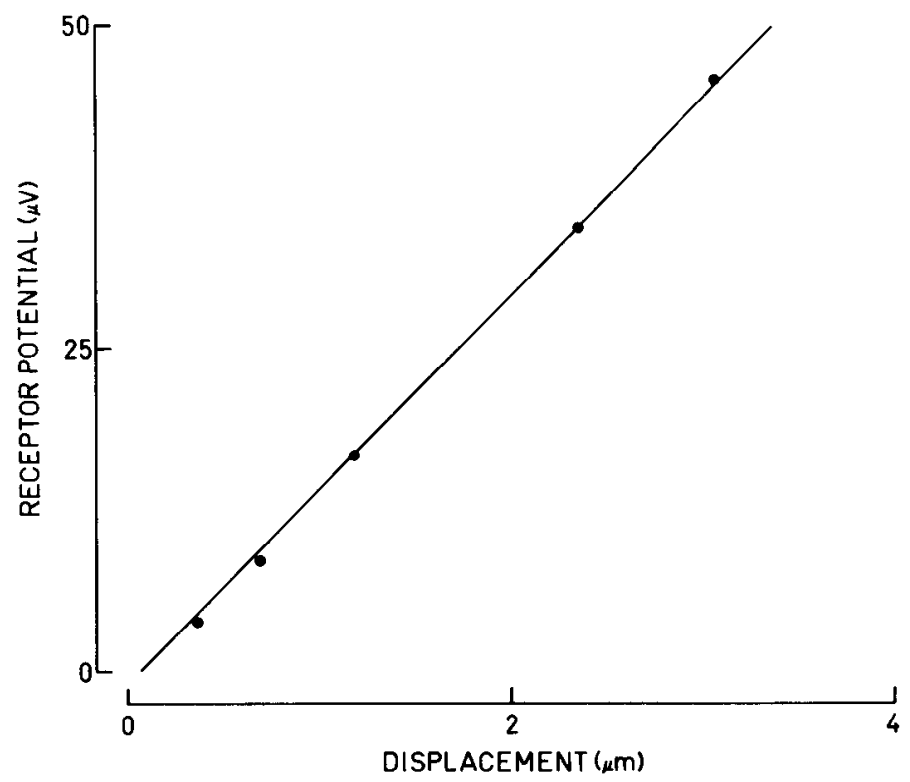

Figure 3. The amplitude of the receptor potential was a linear function of the input displacement for small movements. In this experiment the spine was held at a fixed mean position while varying amplitudes of a $1-\mathrm{Hz}$ sinusoidal modulation of position around the mean were applied, and the resulting receptor potential was measured. The upper limit to the amplitude of displacement was imposed by the threshold for action potential occurrence. The best fitting linear relationship gives a gain of approximately $15 \mathrm{~V} / \mathrm{m}$. 
necessity of remaining below the threshold for action potential production. No sign of nonlinearities, such as rectification or saturation, could be observed.

The frequency response for transduction was measured by varying the frequency of the sinusoidal stimulus. The mean position was held constant during these measurements. Figure 4 shows a typical Bode plot of the frequency response obtained in this way. At low stimulating frequencies the gain of the response was independent of frequency, with no significant phase shift between position and receptor potential. As the frequency rose above about $1 \mathrm{~Hz}$, there was a progressive decrease in gain and a phase lag behind the stimulus. This finding was expected because the voltage was conducted to the recording electrode along an axonal cable. To test if these changes were the result of cable properties, the data were fitted by an equation for the frequency response during conduction along an infinite cable with closed ends (Jack et al., 1975). This gave a good fit to the data, as shown in Figure 4.

The parameters in the equation for the frequency response of a passive axonal cable are the membrane time constant, cable length constant, and length of cable. In addition, the gain of transduction and the recording attenuation are necessary to give the low frequency asymptotic gain. Of these, the only parameters which could be estimated independently of the frequency response were the length constant and the length of the cable. To determine the length constant, the recording electrode was lowered to increase the length of the nerve resting in the tissue fluid instead of in the insulating oil. The amplitude of the receptor potential in response to a $1-\mathrm{Hz}$ sinusoidal movement was recorded before and after a length of nerve was lowered into the saline. This length was measured using a microscope graticule. Twenty measurements were made on 14 different preparations, and the range of length changes was 100 to $225 \mu \mathrm{m}$. The determined length constant of the axonal cable was $131 \pm 28 \mu \mathrm{m}$ (mean \pm standard deviation).

The receptor frequency response was measured 10 times using a different preparation for each set of experiments. The length of nerve from the recording electrode to the sensory cell in the basc of the spine was estimated in each case by measurement with a microscope graticule. The ratio of axonal length to length constant was calculated from the mean value of the length constant, then the asymptotic gain and membrane time constant were fitted to the experimental frequency response.
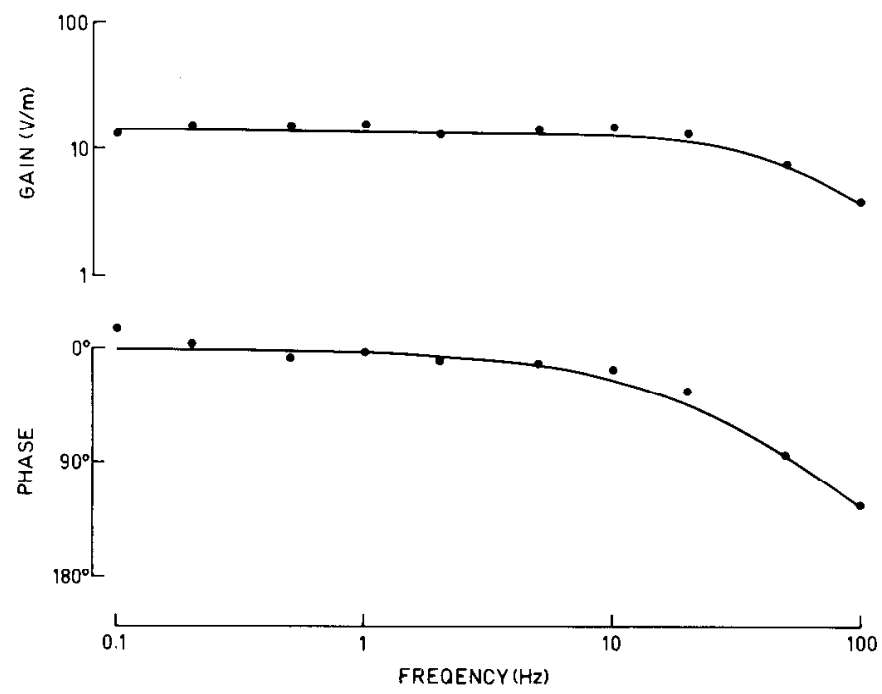

Figure 4. The frequency response function of the receptor potential in response to sinusoidal stimulation over three decades of frequency. The fitted line is the predicted frequency response function for a passive axonal cable of infinite length.
The mean length of nerve from recording site to the base of the spine was $698 \pm 92 \mu \mathrm{m}$, and the mean values of the gain at low frequency and the membrane time constant were $710 \pm$ $198 \mathrm{~V} / \mathrm{m}$ and $1.06 \pm 0.39 \mathrm{msec}$, respectively. Since action potentials recorded in this preparation were 15 to $20 \mathrm{mV}$ in amplitude, it was assumed that the recording arrangement gave an attenuation of three to five relative to the intracellular potential. If this also applied to the receptor potential the gain of transduction in the sensory dendrite would be about 2500 to $3500 \mathrm{~V} / \mathrm{m}$. Movements of approximately $3 \mu \mathrm{m}$, which were just subthreshold, would, therefore, be expected to cause receptor potential amplitudes of 7.5 to $10.5 \mathrm{mV}$, which seems reasonable.

After accounting for the cable properties of the axon, the receptor potential was a linear representation of spine position over at least three decades of frequency with no evidence of adaptation. The upper frequency limit of these measurements was imposed by the cable properties of the axon which reduced the amplitude below the noise level at frequencies above 100 $\mathrm{Hz}$. The lower frequency limit was imposed by the $1 / f$ noise, which made the task of averaging impossibly long below about $0.1 \mathrm{~Hz}$

The dynamic properties of the threshold. To examine the threshold characteristics, a series of experiments were conducted in which the spine was held at a fixed mean position while the amplitude of a sinusoidal movement was increased until just subthreshold; that is, no action potentials occurred during several minutes of observation. The stimulating frequency was then changed and the new threshold amplitude measured again, until a plot was obtained of threshold movement as a function of frequency. The receptor potential was also measured during these experiments, and its relationship to the input movements is described in Figure 4. For simplicity the threshold is plotted versus movement in Figure 5, although a similar diagram could be obtained of threshold versus receptor potential amplitude after correcting for the cable properties of the axon.

As illustrated in Figure 5, the threshold amplitude decreased with increasing frequency and the relationship was linear on a $\log$ amplitude versus $\log$ frequency plot. This corresponds to a negative power law or fractional integrator process in which the fractional exponent is given by the slope of the line. This experiment was repeated on seven different preparations, and the mean exponent was $-0.39 \pm 0.09$ with an average intercept at $1 \mathrm{~Hz}$ of $1.74 \pm 1.25 \mu \mathrm{m}$ displacement. This relationship is exactly the inverse of the power law of sensory transduction measured between movement and action potentials (French and Kuster, 1981). The threshold data suggest that if a constant amplitude sinusoid is applied to the spine the response should increase with frequency by a power law with an exponent of about 0.4 , and that is the result obtained from the frequency response measurements on action potential signals. Therefore, these threshold measurements, in which no action potentials were occurring, could predict the behavior when the preparation was firing many action potentials.

Intracellular recordings. On a few occasions it has been possible to insert a microelectrode into the cell body of the tactile spine sensory neuron. Although these observations are preliminary they have confirmed two of the findings made here by extracellular recordings. The time constant of the cell membrane, measured by injecting a small current step and observing the membrane potential, was determined to be approximately $1 \mathrm{msec}$. This agrees with the value obtained from the frequency response of the axonal cable. When a suprathreshold step of current was injected into the cell body, it fired a rapidly adapting burst of action potentials, in the same manner as has been shown for a step in position (French et al., 1972). This suports the idea that it is the encoder and not the receptor potential which is responsible for the dynamic behavior of this receptor. 


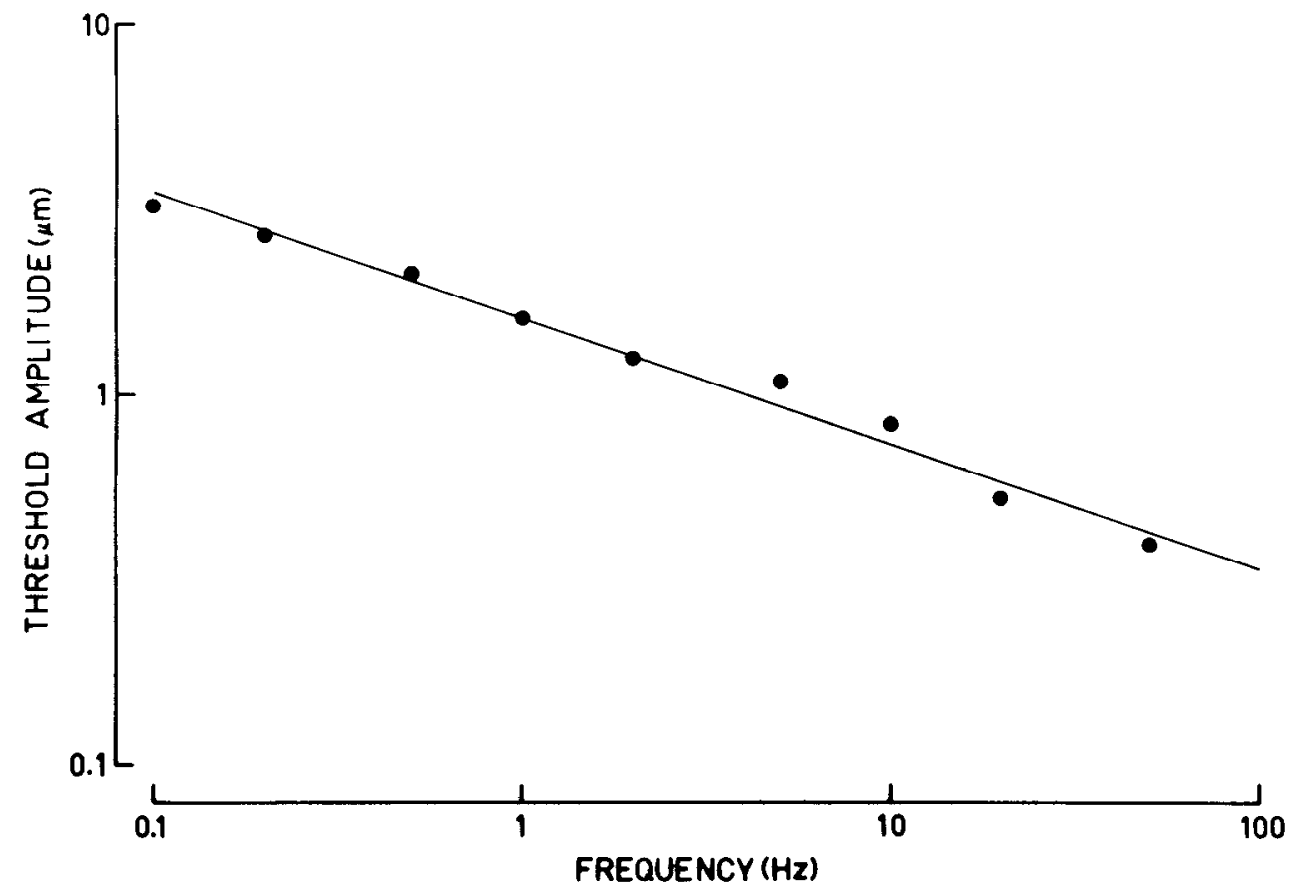

Figure 5. The threshold amplitude of movement to produce action potentials in the tactile spine. The spine was held at a fixed mean position, and the amplitude of the sinusoid was adjusted to be just below threshold at each frequency. The data are plotted as log amplitude versus log frequency of stimulation and are well fitted by a linear function of slope -0.34 , corresponding to a power law, or fractional integration function in time.

\section{Discussion}

In these experiments the receptor potential gave a faithful representation of tactile spine position for subthreshold movements. Such movements might be considered too small to give a useful measure of the receptor potential compared to when the receptor is firing action potentials. However, the strong frequency dependence of the threshold in this neuron means that movements could be applied at low frequencies which were relatively large, compared to those which have been used to study the action potential response. The threshold for sinusoidal movement at $0.1 \mathrm{~Hz}$ was at least $3 \mu \mathrm{m}$, whereas a random stimulus of amplitude as low as $0.5 \mu \mathrm{m}$ root mean square has been used to measure the frequency response of the action potential signal over a range of 0.1 to $100 \mathrm{~Hz}$ (French and Kuster, 1981). 'This was possible because high frequency components in a random signal maintain the firing of action potentials, but these are also modulated by low frequency components which by themselves would be too small to elicit activity. The frequency responses obtained with small random signals were similar to those with movements of up to 20 times larger. Therefore, the movements used here at low frequencies were of comparable amplitude to those which have been used to observe the overall frequency response.

The frequency range of the measurements was restricted by noise and cross-talk considerations, but there was no evidence of increasing response with frequency within the range of 0.1 to $100 \mathrm{~Hz}$. In contrast, the action potential response increases by a power law relationship over this entire range (French and Kuster, 1981). If this frequency sensitivity was a property of the receptor potential, it should have been clearly evident here.

It might be argued that larger movements than those used here, particularly with action potentials occurring, could cause adaptation of the receptor potential by activation of an electrogenic pump or a $\mathrm{Ca}^{2+}$-activated $\mathrm{K}^{+}$conductance. Such mechanisms have been observed in crayfish stretch receptors and muscle spindles (Nakajima and Onodera, 1969a; Husmark and Ottoson, 1971a, b; Ottoson, 1974), and there is preliminary evidence that they are present in the tactile spine preparation (A. S. French, manuscript in preparation). However, it seems unlikely that these effects play a large part in the dynamic properties of the receptor. If action potentials had a strong effect on the receptor potential, then the threshold relationship of Figure 5, obtained without any action potentials, would be very different to the frequency response function obtained when the receptor is firing briskly. Instead they are completely complementary. The logical conclusion is that the dynamic properties of the threshold itself are responsible for the rapidly adapting behavior of this receptor.

Fractional differentiation and action potential encoding. The fractional differentiation seen when the tactile spine is stimulated with step functions, sinusoidal stimuli, or pseudorandom movements (French et al., 1972; French, 1980) is found in other sensory receptors, including arthropod mechanoreceptors (Bohnenberger, 1981), mammalian muscle spindles (Hunt and Wilkinson, 1980), and photoreceptors (Thorson and Biederman-Thorson, 1974). This behavior is apparently linear, since the response to a sinusoidal stimulus is a sinusoid of the same frequency differing only in amplitude and phase. 'Therefore, most theories have concentrated upon linear physical processes which might produce fractional differentiation. One possibility would be that there are many contributing processes with different exponential time courses and that the total response is an average of these, which is well approximated by a power law curve. This idea was formalized into a quantitative theory to explain phototransduction in Limulus (Thorson and Biederman-Thorson, 1974). However, a disadvantage of this theory is that fractional differentiation has been followed over wide frequency ranges in some receptors, such as the five decades of frequency used by Chapman et al (1979), and this would require a similarly wide range of exponential time constants. Another linear mechanism is the traveling wave, or diffusion type of behavior, which can produce semidifferentiation, so that the fractional exponent is 0.5 . This type of process has been suggested to occur in the tubular bodies of insect cuticular mechanoreceptors (French and Sanders, 1981; French and Kuster, 
1982). One simple nonlinear mechanism which has been proposed is that of a power law spring connected to a viscous element in a mechanoreceptor (Brown and Stein, 1966).

All of these proposals involve events occurring in receptors before action potential encoding. As the results presented here show, it is more probable that fractional differentiation arises during encoding, which is a grossly nonlinear process. However, all of the simple models of encoders give frequency response functions which are either independent of stimulus frequency or reduce their response with increasing frequency (Stein et al., 1972; Holden, 1976a). The more complete model of Hodgkin and Huxley for the squid giant axon produces a steady firing in response to a constant current input, although the current must rise sufficiently rapidly to prevent accomodation to the subthreshold stimulus (Hodgkin and Huxley, 1952). The frequency response of the Hodgkin-Huxley model is difficult to characterize because of the phase locking which it gives to repetitive stimuli (Holden, 1976b).

The dynamic characteristics of the threshold in amphibian myelinated fibers have been linked to the $\mathrm{Na}^{+}$inactivation term of the Hodgkin-Huxley equations (Vallbo, 1964b), and in theoretical calculations it was shown that, although changes in both $\mathrm{Na}^{+}$and $\mathrm{K}^{+}$permeabilities could alter the dynamic behavior of the threshold, it was most effectively controlled by changes in $\mathrm{Na}^{+}$inactivation (Frankenhaeuser and Vallbo, 1965). More recently, it has been suggested that differences in several ionic currents, including the regenerative $\mathrm{Na}^{+}$current, delayed $\mathrm{K}^{+}$current, and the $\mathrm{Ca}^{2+}$-activated $\mathrm{K}^{+}$current, must be combined to account for the differences in adaptation of lobster stretch receptors (Gestrelius et al., 1981).

The results presented here seem to eliminate the simpler linear models of fractional differentiation and locate the phenomenon in the action potential encoder of the tactile spine. The exact mechanism by which this complex nonlinear process gives rise to the apparently linear power law behavior will probably have to await better experimental techniques, including intracellular recording and voltage clamping of the sensory cell.

\section{References}

Bernard, J., J. C. Guillet, and J. P. Coillot (1980) Evidence for a barrier between blood and sensory terminal in an insect mechanoreceptor. Comp. Biochem. Physiol. 67A: 573-579.

Bohnenberger, J. (1981) Matched transfer characteristics of single units in a compound slit sense organ. J. Comp. Physiol. 142: 391-402.

Boyd, I. A., M. H. Gladden, and J. Ward (1977) The contribution of intrafusal creep to the dynamic component of the Ia afferent discharge of isolated muscle spindles. J. Physiol. (Lond.) 273: 27-28P.

Brown, M. C., and R. B. Stein (1966) Quantitative studies on the slowly adapting stretch receptor of the crayfish. Kybernetik 3: 175-185.

Buno, W., L. Monti-Bloch, A. Mateos, and P. IIandler (1981) Dynamic properties of cockroach cercal "threadlike" hair sensilla. J. Neurobiol. 12: $123-141$.

Catton, W. T., and N. Petoe (1966) A visco-elastic theory of mechanoreceptor adaptation. J. Physiol. (Lond.) 187: 35-49.

Chapman, K. M., and R. S. Smith (1963) A linear transfer function underlying impulse frequency modulation in a cockroach mechanoreceptor. Nature 197: 699-700.

Chapman, K. M., J. L. Mosinger, and R. B. Duckrow (1979) The role of distributed viscoeleastic coupling in sensory adaptation in an insect mechanoreceptor. J. Comp. Physiol. 131: 1-12.

Chesler, M., and C. R. Fourtner (1981) Mechanical properties of a slow muscle in the cockroach. J. Neurobiol. 12: 391-402.

Eyzaguirre, C., and S. W. Kuffler (1955) Processes of excitation in the dendrites and in the soma of single isolated sensory nerve cells of the lobster and crayfish. J. Gen. Physiol. 39: 87-119.

Frankenhaeuser, B., and A. B. Vallbo (1965) Accommodation in myelinated nerve fibres of Xenopus laevis as computed on the basis of voltage clamp data. Acta Physiol. Scand. 63: 1-20.
French, A. S. (1980) Sensory transduction in an insect mechanoreceptor: Linear and nonlinear properties. Biol. Cybernetics 38: 115-123.

French, A. S., and J. E. Kuster (1981) Sensory transduction in an insect mechanoreceptor: Extended bandwidth measurements and sensitivity to stimulus strength. Biol. Cybernetics 42: 87-94.

French, A. S., and J. E. Kuster (1982) The effects of temperature on mechanotransduction in the cockroach tactile spine. J. Comp. Physiol. 147: 251-258.

French, A. S., and E. J. Sanders (1981) The mechanosensory apparatus of the femoral tactile spine of the cockroach, Periplaneta americana. Cell Tissue Res. 219: 53-68.

French, A. S., A. V. Holden, and R. B. Stein (1972) The estimation of the frequency response function of a mechanoreceptor. Kybernetik 11: $15-23$.

Gestrelius, S., W. Grampp, and L. Sjolin (1981) Subthreshold and nearthreshold membrane currents in lobster stretch receptor neurones. J. Physiol. (Lond.) 310: 191-203.

Hodgkin, A. L., and A. F. Huxley (1952) A quantitative description of membrane current and its application to conduction and excitation in nerve. J. Physiol. (Lond.) 117: 500-544.

Holden, A. V. (1976a) Models of the Stochastic Activity of Neurones, Springer-Verlag, Heidelberg.

Holden, A. V. (1976b) The response of excitable membrane models to a cyclic input. Biol. Cybernetics 21: 1-7.

Hunt, C. C., and R. S. Wilkinson (1980) An analysis of receptor potential and tension of isolated cat muscle spindles in response to sinusoidal stretch. J. Physiol. (Lond.) 302: 241-262.

Husmark, I., and D. Ottoson (1971a) The contribution of mechanical factors to the early adaptation of the spindle response. J. Physiol. (Lond.) 212: 577-592.

Husmark, I., and D. Ottoson (1971b) Ionic effects on spindle adaptation. J. Physiol. (Lond.) 218: 257-269.

Ito, F., and Y. Komatsu (1979) Calcium-dependent regenerative responses in the afferent nerve terminal of the frog muscle spindle. Brain Res. 175: 160-164.

Jack, J. J. B., D. Noble, and R. W. Tsien (1975) Electric Current Flow in Excitable Cells, Clarendon Press, Oxford.

Katz, B. (1950) Depolarization of sensory terminals and the initiation of impulses in the muscle spindle. J. Physiol. (Lond.) 111: 261-282.

Loewenstein, W. R. (1956) Excitation and changes in adaptation by stretch of mechanoreceptors. J. Physiol. (Lond.) 133: 588-602.

Loewenstein, W. R. (1959) The generation of electric activity in a nerve ending. Ann. N. Y. Acad. Sci. 81: 367-387.

Matthews, P. B. C., and R. B. Stein (1969) 'I'he sensitivity of muscle spindle afferents to sinusoidal stretching. J. Physiol. (Lond.) 200: $723-743$.

McIver, S. B. (1975) Structure of cuticular mechanoreceptors of arthropods. Annu. Rev. Entomol. 20: 381-397.

Mendelson, M., and W. R. Loewenstein (1964) Mechanisms of receptor adaptation. Science 144: 554-555.

Nakajima, S., and K. Onodera (1969a) Adaptation of the generator potential in the crayfish stretch receptors under constant length and constant tension. J. Physiol. (Lond.) 200: 187-204.

Nakajima, S., and K. Onodera (1969b) Membrane properties of the stretch receptor neurones of crayfish with particular reference to mechanisms of sensory adaptation. J. Physiol. (Lond.) 200: 161-185.

Nijenhuis, E. D., and D. Dresden (1952) A micromorphological study of the sensory supply of the mesothoracic leg of the american cockroach, Periplaneta americana. Proc. K. Ned. Akad. Wet. 55: 300-310.

Ottoson, D. (1974) Adaptive properties of crayfish stretch receptor neurons. In Symposium in Mechanoreception, Schwartzkopf, ed., pp. 401-412, Westdeutscher Verlag, Opladen.

Stein, R. B., A. S. French, and A. V. Holden (1972) The frequency response, coherence and information capacity of two neuronal models. Biophys. J. 12: 295-322.

Thorson, J., and M. Biederman-Thorson (1974) Distributed relaxation processes in sensory adaptation. Science 183: 161-172.

Vallbo, A. B. (1964a) Accommodation of single myelinated nerve fibres from Xenopus laevis related to type of end organ. Acta Physiol. Scand. 61: 413-428.

Vallbo, A. B. (1964b) Accommodation related to inactivation of the sodium permeability in single myelinated nerve fibres from Xenopus laevis. Acta Physiol. Scand. 61: 429-444. 